\title{
The Student Counseling Virtual Pamphlet Collection: An innovative resource
}

\author{
ROBERT C. HSIUNG \\ University of Chicago, Chicago, Illinois
}

\begin{abstract}
Many university student counseling centers are now adapting pamphlets for the World-Wide Web, creating what the author refers to as virtual pamphlets. The author searched 52 university student counseling center Web sites, uncovered 200 virtual pamphlets, and brought them together as the Student Counseling Virtual Pamphlet Collection. In just a few months, there are already multiple indications that this innovative resource is considered valuable. Moreover, this paradigm can be applied, and different virtual pamphlet collections developed, to promote the education of clients who receive other mental health, general medical, or even non-health-related services.
\end{abstract}

Presented here is the Student Counseling Virtual Pamphlet Collection, an innovative resource for university student counseling centers. By virtual pamphlets, we refer to information on the World-Wide Web (or, more simply, "the Web") that serves the same function as pamphlets printed on paper. Such Web "pages" (units of information) are virtually, but not quite, pamphlets, since they exist only in electronic form (although users may, of course, print them out). Many virtual pamphlets start out as actual pamphlets and are only later adapted for the Web.

Our focus is on university student counseling, by which we mean the counseling of undergraduates and graduate students. The concept of virtual pamphlet collections easily generalizes, however, to the delivery of many other types of services. We compare actual and virtual pamphlets, review the methods by which the Student Counseling Virtual Pamphlet Collection was developed, describe the collection itself, and discuss preliminary evaluative data.

\section{Actual Versus Virtual Pamphlets}

There is a long tradition of university student counseling centers offering actual pamphlets to students. At the University of Chicago Student Counseling and Resource Service (SCRS), for example, we produce "Substance Abuse" ourselves and purchase, among others, "Stress in College: Stretching the Rubber Band" and "Beating Back the Blues: Dealing with Depression" from the American College Health Association, "Depression" and "Substance Abuse" from the American Psychiatric Association, "Marijuana: Personality \& Behavior" and "Cocaine: Waking Up To A Nightmare" from the Do It Now Foundation.

There are two overlapping rationales for providing such pamphlets. First, they educate students. "Beating Back the Blues," for example, describes the various symp-

R. C. Hsiung is Deputy Medical Director of the Student Counseling and Resource Service at the University of Chicago, where he is also affiliated with the Department of Psychiatry. He may be reached at 5737 S. University Ave., Chicago, IL 60637-1507 (e-mail:r-hsiung@ uchicago. edu). toms of depression. Some, such as "Depression," even include bibliographies that introduce students to the literature. This attention to education is especially fitting in a university setting.

Pamphlets also help students to care more effectively for themselves. "Stress in College," for example, discusses both short-term and long-term ways to handle stress. Not only may these techniques help students with the specific problems at hand, but they may also promote an overall attitude of self-care. Development along these lines is also particularly relevant with a population of late adolescents and young adults.

Compared with actual pamphlets, virtual pamphlets have both advantages and disadvantages. Most virtual pamphlets include information about services at the originating, distant universities that does not apply to students elsewhere. This is ameliorated in the Student Counseling Virtual Pamphlet Collection by a reminder, which stays visible in a separate "frame" (portion of the window), about the local university student counseling center.

Copyright is not an issue because the virtual pamphlets are not copied, but only linked to. "Links" are special text that users can click on to move from one Web page to another, like high-tech citations. Neither is there any more risk of malpractice with virtual pamphlets than with actual ones. Providing pamphlets of any sort is an educational service, not a clinical one.

It is possible for students to "visit" (connect to) virtual pamphlets, but never actually meet with counselors. If the virtual pamphlets do not help them, however, those students will remain potential clients of their own centers. Meanwhile, virtual pamphlets are available 24 hours a day, 7 days a week, which counseling centers cannot rival, and the problems for which pamphlets might be of use often surface after business hours. Some students are also uncomfortable with the possibility of being seen visiting their counseling centers. They can connect to virtual pamphlets, however, without being observed.

Virtual pamphlets are more current. Since they are linked to, rather than copied, as soon as the master files 
are updated by the originating centers, those new versions are what users will receive when they connect. The centers linking to them need do nothing.

The most obvious advantage of the Student Counseling Virtual Pamphlet Collection is probably its bringing together in one place virtual pamphlets scattered around the World-Wide Web. Without such a centralized directory, efforts to link to virtual pamphlets would likely be hit-or-miss.

Virtual pamphlets clearly entail great savings. They do not need to be drafted, revised, approved, and printed, or even purchased, by centers that link to them. Also, there is no wastage of obsolete virtual pamphlets, as there would be with actual pamphlets, when revisions are made. The training or hiring of staff to develop and maintain Web pages can be an expense, but evolving software is making it easier to create and manage Web pages, Web page-editing proficiency is becoming more commonplace, and it is also possible to share human resources with parent universities.

The number of offerings in the Student Counseling Virtual Pamphlet Collection is an order of magnitude greater than that at the SCRS and probably most other university student counseling centers. Virtual pamphlets from university student counseling centers are also targeted to university students. Commercially available pamphlets can be informative, but may not address more specialized issues such as "Long Distance Relationships," from the University of Missouri-Rolla (http://www. umr.edu/ counsel/ long.html). University student counseling centers are also able to cover more obscure or timely subjects such as "Cult Awareness," from Purdue University (gopher:// oasis.adpc.purdue.edu:2525/00/student $/ \mathrm{mental} / \mathrm{cm} 340$ ), or "Roofies" (Rohypnol), from the University of Massachusetts at Lowell (http://aspen.uml.edu/www/counseling/ roofies.html).

While the quality of the information available on the Internet is generally quite variable, virtual pamphlets from university student counseling centers are reliable, produced, as they are, by qualified professionals. This does not mean that different university student counseling centers do not take different approaches: a wide variety of virtual pamphlets is available. On the topic of eating disorders, for example, "Body Acceptance and Eating Disorders," from the University of Florida (http://www.ufsa.ufl.edu/Counsel/Body.htm), offers quizzes to evaluate preoccupation with food and satisfaction with one's own body, "Eating Disorders: Myths and Campus Resources," from George Mason University (http://web.gmu.edu/departments/csdc/eat. html), lists different "myths" about eating disorders, and "Eating Disorders," from the University of Massachusetts at Lowell (http://aspen.uml.edu/www/counseling/eatdis. $\mathrm{html}$ ), includes a more traditional list of behaviors of concern. There is no one single correct solution to a given problem. Different alternatives will appeal to and be helpful for different students; the more options that are available, the greater the number of students who will be reached.
Certain university student counseling centers have special areas of interest or expertise, and other centers can then benefit from their especially comprehensive virtual pamphlets (or sets of virtual pamphlets). For example, SUNY at Buffalo presents several pamphlets pertaining to rape, including "Date and Acquaintance Rape" (http://wings.buffalo.edu/student-life/ccenter/Relationships/Violence/rape.acq) and "Sexual Assault and Rape: Advice for Men" (http://wings.buffalo.edu/student-life/ ccenter/Relationships/Men/advice.men).

One view could be that offering the Student Counseling Virtual Pamphlet Collection at a university cedes to outsiders partial control over the information provided to the students there. We welcome, however, the collaborative sharing of virtual pamphlets as a form of division of labor. Work in a university student counseling center can be isolating. One is hardly in the mainstream of campus life, and many centers are small, making for few on-site colleagues. If university student counselors develop virtual pamphlets that are used by their colleagues, the sense of community between them will be enhanced.

\section{Development of the Student Counseling Virtual Pamphlet Collection}

Identification of student counseling center Web sites. The SCRS has been active in the provision of mental health information on the Web (Grohol, 1996; Hsiung, 1996b, 1996c, 1996d; Hu, 1996; Klein, 1996; Knowlton, 1996; Lim \& Hsiung, 1996). This has included the development of the Web "site" (set of Web pages) that describes the services provided by the SCRS (Hsiung, 1996d, http://uhs.bsd.uchicago.edu/scrs/scrs.html). This led to our becoming aware of David L. Gilles-Thomas's compilation of Student Counseling Centers on the Internet, which included a list of university student counseling center Web sites, and we adapted his list for the Web (http://wings.buffalo.edu/student-life/ccenter/centers. html). We also belong to the Counseling Center Directors electronic discussion group, started by Donald Chezik and overseen next by Ralph Roberts and now by Paul Maierle, on which new university student counseling center Web sites are sometimes announced.

From these sources, 55 university student counseling center Web sites were located. One university was in Australia, and the rest were in the U.S. Three sites, including the Australian one, appeared to be "down" or in transition and were not accessible. Since there are 3,688 colleges and universities in the U.S. ("The Nation," 1996), it would not be surprising if more than this $1.5 \%$ had counseling center Web sites without our becoming aware of them. For the Student Counseling Virtual Pamphlet Collection to be of value, however, it need not be exhaustive.

Selection of virtual pamphlets. We visited the 52 university student counseling center Web sites that were operational and searched them for virtual pamphlets. This searching was decidedly low tech and involved no "search engines" (Web-page databases). We started at the centers' "home" (introductory) pages and systematically followed each of the links to other pages at those sites. We 
added to the collection each page (or, in the case of wideranging pages, each section of a page) that (1) was developed by that center and (2) presented information of general interest about either (a) psychological problems or (b) treatments. Pages that were copies of material from other centers were not included, nor were those that focused on specifics of the originating institutions. No attempt was made to choose between different virtual pamphlets covering the same subjects.

Twenty-three (44\%) of the university student counseling center Web sites included virtual pamphlets, and the latter turned out to number exactly 200.

Any Web browser could have been used to explore the counseling center Web sites, and any word processor could have been used to construct the actual Student Counseling Virtual Pamphlet Collection Web page, but we used Netscape Navigator and Microsoft Word, respectively.

\section{Description of the Student Counseling Virtual Pamphlet Collection}

The address or uniform resource locator (URL) of the Student Counseling Virtual Pamphlet Collection is http://uhs.bsd.uchicago.edu/scrs/vpc/vpc.html.

Web page structure. The Web browser window (see Figure 1) is divided into two frames. The main, upper, one is used to display the virtual pamphlet collection and the virtual pamphlets themselves. The smaller, lower, one shows information about the local university student counseling center. Both frames stay visible at all times, so the local information is always close at hand, no matter where in the world the virtual pamphlets that are displayed are from (this is analogous to the superimposition of the local temperature on a national weather forecast).

Any Web browser can be used to access the Student Counseling Virtual Pamphlet Collection, but only more recent versions (such as Netscape Navigator 2.0 and on) are able to divide the window into frames. Older versions can still connect, but will not show the local information frame.

At the top of the collection are links to background information and instructions on how to link to the page and to submit a virtual pamphlet for inclusion. Next is a reminder that the virtual pamphlets put out by other universities may refer to services available only to students at those universities, and that other students should, of course, contact their own university student counseling centers instead. After that is a list of the 18 topics into which we have grouped the virtual pamphlets. The topics and the number of virtual pamphlets in each are listed in Table 1.

The list of topics, which serves as an index, is followed by the sections devoted to each topic. The name of

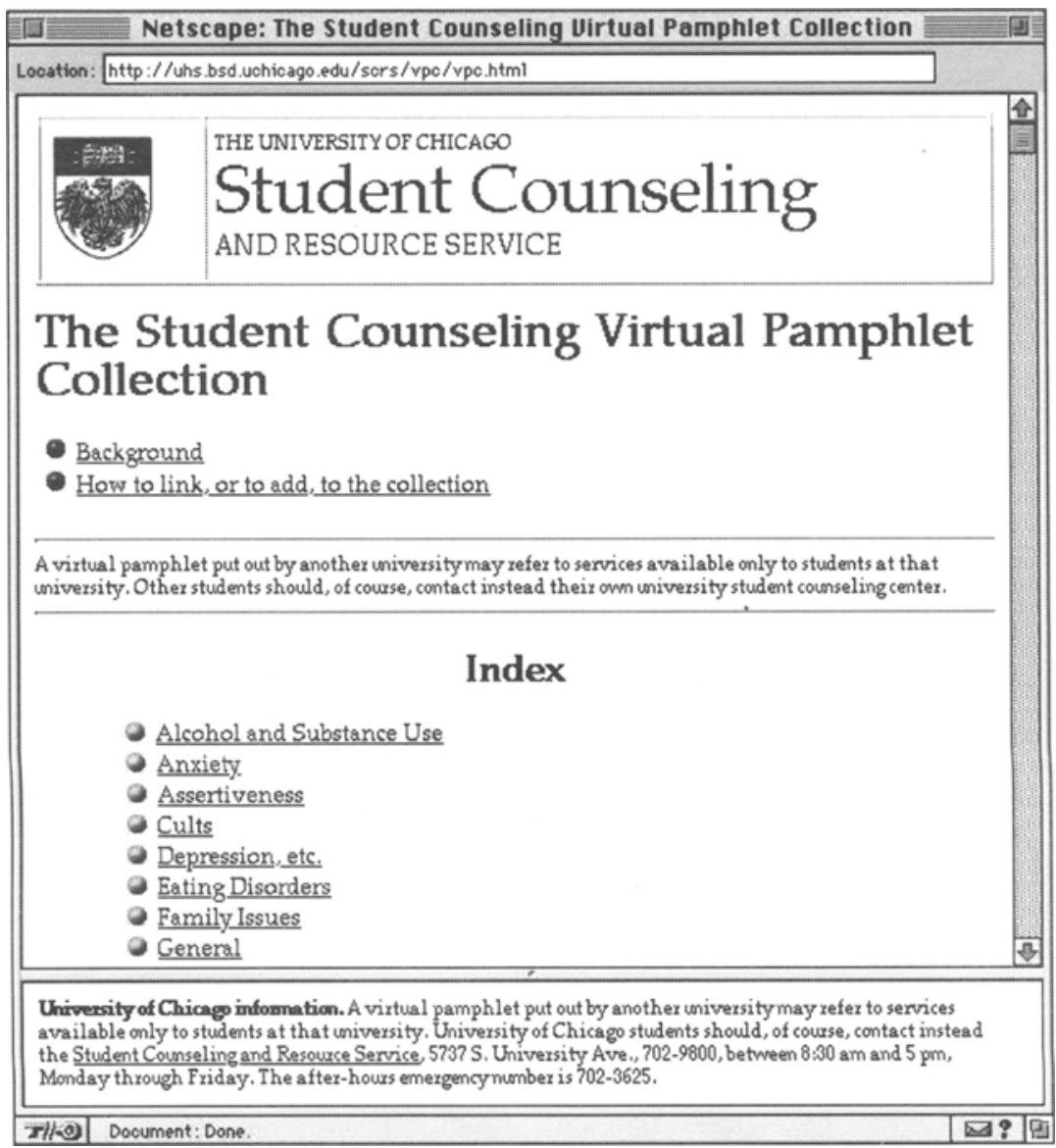

Figure 1. The Student Counseling Virtual Pamphlet Collection, at http://uhs.bsd. uchicago.edu/scrs/vpc/vpc.html, as seen when the user first connects to it. 
each topic is a link to the corresponding section. The user clicks on a topic and is taken (in the main frame) to the corresponding section. Each section lists the relevant virtual pamphlets and the originating universities. As above, the name of each virtual pamphlet is a link to that virtual pamphlet. The user clicks on a name and is taken to that virtual pamphlet.

Evaluative data. The Student Counseling Virtual Pamphlet Collection debuted in October 1996. A link to the Student Counseling Virtual Pamphlet Collection was added to the SCRS home page. Gilles-Thomas and the Counseling Centers Directors group were informed by e-mail of the new resource. The collection was also entered into the databases of the various Web-search services.

We have received a number of positive comments, many from directors of university student counseling centers. Another indication of utility is popularity, one measure of which is how often the page is accessed, and that statistic is increasing, from 357 in October 1996 to 415 in November and 534 in December. Moreover, several university student counseling centers have already linked to the collection, and some have taken advantage of the ability to customize it with their own local information.

\section{Development of Other Virtual Pamphlet Collections}

The process of developing a virtual pamphlet collection can easily be adapted to other mental health, general medical, or even non-health-related services. Prior to undertaking such an endeavor, however, two basic questions should be considered.

First, are virtual pamphlets available? In the area of university student counseling, they are, because university student counseling centers are relatively likely to have Internet access and because the information they provide is not proprietary. College career counseling might

Table 1

Number of Virtual Pamphlets in the Student Counseling Virtual Pamphlet Collection, by Topic

\begin{tabular}{lc}
\multicolumn{1}{c}{ Topic } & $\begin{array}{c}\text { Number of } \\
\text { Virtual Pamphlets }\end{array}$ \\
\hline Alcohol and substance use & 12 \\
Anxiety & 5 \\
Assertiveness & 6 \\
Cults & 1 \\
Depression, etc. & 26 \\
Eating disorders & 8 \\
Family issues & 5 \\
General & 15 \\
Relationships & 15 \\
Sexual assault & 13 \\
Sexual harassment & 1 \\
Sexual orientation & 4 \\
Sleep & 1 \\
Stress & 9 \\
Study skills & 38 \\
Time management & 13 \\
Traumatic events & 3 \\
For concerned others & 25 \\
Total & 200 \\
\hline
\end{tabular}

be similar in these respects, with virtual pamphlets on writing résumés, interviewing, and so forth. On the other hand, stockbrokers might be less inclined to make public the information they have to offer and automobile mechanics might not even have Web sites.

Second, how will the collection be focused? The more focused the collection, the more manageable the collecting. One type of focus is on a topic. Ours was university student counseling. Counseling, in general, would be much more daunting to tackle. Another type of focus is on the intended audience. Ours was university students and concerned others. It did not include other university student counselors, for which a different sort of virtual pamphlet would have been more appropriate. A third type of focus is on particular virtual pamphlet providers. The virtual pamphlets developed by student counseling centers differ, for example, from those produced by other kinds of organizations (such as the American Psychological Association-http://www.apa.org/—or the National Alliance for the Mentally Ill-http://www.nami.org/) or by individual clinicians or clients. We were fortunate to be able to have available a listing of student counseling centers on the Web, our virtual pamphlet providers.

\section{Conclusion}

The Student Counseling Virtual Pamphlet Collection is a significant new type of resource for university students and university student counseling centers, one that offers many advantages over more traditional racks of pamphlets printed on paper. Moreover, the process of developing a virtual pamphlet collection can easily be adapted to a broad range of other services.

\section{REFERENCES}

GROHOL, J. (1996). What's available on-line for mental health professionals. Psychiatric Times, 13(9), 51-52.

Hsiung, R. C. (1996a, October). Psychopharmacology on the WorldWide Web. Paper presented at the American Psychiatric Association Institute on Psychiatric Services, Chicago.

Hsiung, R. C. (1996b, May). Psychopharmacology tips on the WorldWide Web. Paper presented at the American Psychiatric Association Annual Meeting, New York.

Hsiung, R. C. (1996c, March). Web connections: A practical psychopharmacology resource. Paper presented at the Big 10 Counseling Centers Conference, University Park, PA.

Hsiung, R. C. (1996d, March). Web connections: The University of Chicago Student Counseling and Resource Service. Paper presented at the Big 10 Counseling Centers Conference, University Park, PA.

Hu, R. J. (1996). You need the Net: A pep-talk for the resident or young researcher. Psychiatric Research Report, 12(2), 8-9.

KLEIN, H. E. (1996). Web sites for behavioral health practitioners. Psychotherapy Finances, $22(2), 5$.

KNOWLTON, L. (1996). Computer coverage nets capacity crowds. Psychiatric Times, 13(9), 48-49.

Lim, R., \& Hsiung, R. C. (1996, October). Using the Internet: Research, training and treatment. Paper presented at the American Psychiatric Association Institute on Psychiatric Services, Chicago.

The Nation: Colleges and Universities (1996, September). Chronicle of Higher Education, 43(1), 3.

(Manuscript received October 3, 1996; revision accepted for publication February 10, 1997.) 